\title{
Ensemble Pruning using Spectral Coefficients
}

\author{
Terry Windeatt, Cemre Zor
}

\begin{abstract}
Ensemble pruning aims to increase efficiency by reducing the number of base classifiers, without sacrificing and preferably enhancing performance. In this paper, a novel pruning paradigm is proposed. Two class supervised learning problems are pruned using a combination of first and second order Walsh coefficients. A comparison is made with other ordered aggregation pruning methods, using Multilayer Perceptron base classifiers. The Walsh pruning method is analysed with the help of a model that shows the relationship between second order coefficients and Added Classification Error with respect to Bayes Error.
\end{abstract}

Keywords: Classification, Pattern Analysis, Ensemble Pruning

\section{INTRODUCTION}

The advantage of an ensemble compared to individual classifiers is now well established. However, there are many design issues that remain to be addressed, such as ensemble pruning (sometimes called selection or thinning [1]) which aims to reduce the number of base classifiers without sacrificing performance. The advantages may include enhanced generalisation performance as well as reduced complexity and storage requirements. In general, the selection of an optimum subset of classifiers is computationally expensive and grows exponentially with the number of classifiers. For $\mathrm{N}$ classifiers, an exhaustive search would need to consider $2^{N}-1$ sub-ensembles.

Various pruning techniques based on combinatorial search have been attempted. The simplest is a greedy search based on ordered aggregation such as Margin Distance Minimisation [2] (MDP Section III). Though simple, MDP claims comparable performance with more sophisticated strategies. Other types of pruning methods are based on clustering and on optimisation frameworks [3], such as quadratic integer programming [4]. More recent methods include probabilistic pruning using expectation propagation [5], regularisation of pruned bagging ensembles [6] and constructive approaches [7], that claim no need of pruning. Statistical instance-based sampling [8] is aimed at efficiency rather than accuracy, but may be combined with other pruning methods [9]. In this paper, a novel pruning approach is proposed, which exploits .the spectral coefficients of a binary-to-binary mapping, and is compared with other ordered aggregation methods. A further contribution is the explanation of pruning using the TumerGhosh model [14].

Early work on Walsh functions [10] recognised that Walsh coefficients could be useful for Pattern Recognition applications, but it was much later that their use in ensemble design was first proposed [11]. First order coefficients were computed using spectral summation in [12], and used to select optimal base classifier complexity. In [13], the link was made between Walsh coefficients and the model of Added Classification Error, that is error added to Bayes [14]. In [13], base classifier complexity was varied and second order

Manuscript received Nov. 8, 2011. This work was supported in part by UK Government, EPSRC under grant number E061664/1

T. Windeatt, C. Zor are with the Centre for Vision Speech and Signal Processing, Faculty of Electronics and Physical Sciences, University of Surrey, Guildford Surrey, GU2 7XH, UK (phone: +44-1483-9286; e-mail: t.windeatt@surrey.ac.uk) coefficients were maximised. In contrast, in this paper it is assumed that base classifiers are optimised and on either side of the Bayes boundary. Furthermore second order coefficients are minimised when applied to ordered aggregation pruning.

Section II explains the computation of Walsh coefficients and shows their relationship with the model of Added Classification Error. Ordered aggregation pruning methods, including the proposed Walsh pruning, are described in Section III, with an experimental comparison in Section IV using single hidden-node MLP base classifiers.

\section{WALSH COEFFICIENTS \& ADDED ERROR}

Consider an ensemble framework, in which there are $N$ parallel base classifiers, and $X_{m}$ is the $N$-dimension vector representing the $m$ th training pattern, formed from the decisions of the $N$ classifiers. For a two-class supervised learning problem of $\mu$ training patterns, the target label given to each pattern $X_{m}$ is denoted by $\Omega_{m}=\Phi\left(X_{m}\right)$ where $m=$ $1 \ldots \mu, \Omega_{m} \in\{1,-1\}$ and $\Phi$ is the unknown Boolean function that maps $X_{m}$ to $\Omega_{m}$. Thus the binary vector $X_{m}$ represents the $m t h$ original training pattern

$$
\begin{aligned}
& X_{m}=\left(X_{m 1}, X_{m 2}, \ldots, X_{m N}\right) \\
& \text { where } \quad X_{m i} \in\{1,-1\} \text { is a vertex } \\
& \text { sional binary hypercube. The Walsh trans } \\
& T_{n}=\left[\begin{array}{cc}
T_{n-1} & T_{n-1} \\
T_{n-1} & -T_{n-1}
\end{array}\right], T_{1}=\left[\begin{array}{cc}
1 & 1 \\
1 & -1
\end{array}\right]
\end{aligned}
$$$$
\text { where } X_{m i} \in\{1,-1\} \text { is a vertex in the } N \text { - }
$$

dimensional binary hypercube. The Walsh transform of $\Phi$ is derived from the mapping $\mathrm{T}_{\mathrm{n}}$ and defined recursively as follows

The first and second order spectral coefficients $s_{i}$ and $s_{i j}$ derived from (2) are defined in [10] as

$$
\begin{gathered}
s_{i}=\sum_{m=1}^{\mu}\left(X_{m i} \Omega_{m}\right) \\
s_{i j}=\sum_{m=1}^{\mu}\left(X_{m i} \oplus X_{m j}\right) \Omega_{m}
\end{gathered}
$$

In (3) $S_{i}$ represents the correlation between $\Omega_{m}$ and $X_{m i}$ and $s_{i j}(i, j=1 \ldots N, i \neq j)$ in (4) represents correlation between $\Omega_{m}$ and $X_{m i} \oplus X_{m j}$, where $\oplus$ is defined as -1 iff $X_{m i} \neq X_{m j}$. For third order coefficients, correlation is between $\Omega_{m}$ and $X_{m i} \oplus X_{m j} \oplus X_{m k}$ and higher order follows, but in this paper we restrict ourselves to first and second order spectral coefficients.

Let $n_{a}$ be the number of class -1 patterns for which classifiers $i, j$ disagree

$$
n_{a}=\sum_{m=1}^{\mu}\left(I\left(X_{m i} \neq X_{m j}\right): \Omega_{m}=-1\right)
$$

where $I(\cdot)$ is defined as 1 if pair $i, j$ disagree, otherwise 0 . Similarly $n_{b}$ is the number of class -1 patterns with $i, j$ agreeing, that is $I\left(X_{m i}=X_{m j}\right)$ in (5). Corresponding 
definitions for $n_{c}$ and $n_{d}$ are number of class 1 patterns ( $\Omega_{m}=1$ in (5)) with classifiers $i, j$ disagreeing and agreeing, respectively. According to [15]

$$
s_{i j}=\left(n_{a}+n_{d}\right)-\left(n_{b}+n_{c}\right)
$$

which does not depend on the unspecified patterns. Therefore (6) may be applied to a Boolean function $\Phi$ that is incompletely specified, which would be the case for the realistic machine learning problem, defined in (1).

To understand the coefficient calculation, the following truth table represents an example three variable Boolean function taken from [12].

\begin{tabular}{lrrrr}
\hline $\mathrm{X}_{\mathrm{m}}$ & $\mathrm{X}_{\mathrm{m} 1}$ & $\mathrm{X}_{\mathrm{m} 2}$ & $\mathrm{X}_{\mathrm{m} 3}$ & $\Omega_{\mathrm{m}}$ \\
\hline $\mathrm{X}_{1}$ & 1 & 1 & 1 & 1 \\
$\mathrm{X}_{2}$ & -1 & 1 & 1 & -1 \\
$\mathrm{X}_{3}$ & 1 & -1 & 1 & -1 \\
$\mathrm{X}_{4}$ & -1 & -1 & 1 & 1 \\
$\mathrm{X}_{5}$ & 1 & 1 & -1 & 1 \\
$\mathrm{X}_{6}$ & -1 & 1 & -1 & -1 \\
$\mathrm{X}_{7}$ & 1 & -1 & -1 & -1 \\
$\mathrm{X}_{8}$ & -1 & -1 & -1 & -1 \\
\hline
\end{tabular}

The truth table ordering defines the spectral coefficient ordering [10], which is computed as follows for $T_{3}$

$$
\begin{aligned}
& T_{3}\left[\begin{array}{c}
1 \\
-1 \\
-1 \\
1 \\
1 \\
-1 \\
-1 \\
-1
\end{array}\right]=\left[\begin{array}{c}
-2 \\
2 \\
2 \\
6
\end{array}\right] s_{0} s_{2} \\
& T_{3}=\left[\begin{array}{cccccccc}
1 & 1 & 1 & 1 & 1 & 1 & 1 & 1 \\
1 & -1 & 1 & -1 & 1 & -1 & 1 & -1 \\
1 & 1 & -1 & -1 & 1 & 1 & -1 & -1 \\
1 & -1 & -1 & 1 & 1 & -1 & -1 & 1 \\
1 & 1 & 1 & 1 & -1 & -1 & -1 & -1 \\
1 & -1 & 1 & -1 & -1 & 1 & -1 & 1 \\
1 & 1 & -1 & -1 & -1 & -1 & 1 & 1 \\
1 & -1 & -1 & 1 & -1 & 1 & 1 & -1
\end{array}\right]
\end{aligned}
$$

By comparing the truth table and the transformation matrix in (8), it may be seen that first order coefficients, $s_{1}, s_{2}, s_{3}$ in (7), represent the first order correlation defined in (3), which may be interpreted as the difference between the correct and incorrect classifications. Similarly the second order coefficients, $s_{12}, s_{23}, s_{13}$ in (7), represent second order correlation defined in (4). It is easy to verify the result of applying (3) and (6). For example

$$
s_{1}=5-3=2 \text { and } s_{12}=(4+3)-(1+0)=6 \text {. }
$$

Let's assume that the second pattern $\mathrm{X}_{2}$ is unspecified, so

$$
s_{1}=4-3=1 \text { and } s_{12}=(3+3)-(1+0)=5 \text {. }
$$

Note that in [12] a more complex calculation based on spectral summation is proposed for first order coefficients, and represents a different way of generalisation for unspecified patterns. For those knowledgeable on logic design the spectral coefficients are interpreted on a Karnaugh map in [15].

Fig. 2 shows the two class $\left(\omega_{1}, \omega_{2}\right)$ model of Added Classification Error, restricted to one dimension $(x)$, which was proposed in [14]. The model assumptions are that the distributions are approximated by base classifier outputs and locally monotonic around the Bayes boundary. Furthermore it is assumed that estimation errors are i.i.d. The monotonicity assumption is fairly robust, as noted in [16], since typically boundaries are located in transition regions where the posteriors are not in local extrema. In [14], the i.i.d. assumption is relaxed, as given in (13).

The output of the $j t h$ classifier representing class $\omega_{1}$ is given by $\hat{P}_{j}\left(\omega_{1} \mid x\right)=P_{j}\left(\omega_{1} \mid x\right)+\varepsilon_{1}(x)$ where $P, \hat{P}$ are actual and estimated a posteriori probability distributions as shown in Fig. 2, and $\varepsilon_{1}(x)$ is the estimation error. Similar equation exists for class $\omega_{2}$. Note in Fig. 2 that the estimated probabilities for ith classifier $\hat{P}_{i}\left(\omega_{2} \mid x\right)$ and $\hat{P}_{i}\left(\omega_{1} \mid x\right)$ are omitted for clarity. If $b$ in Fig. 2 is the displacement of the $j$ th classifier boundary $\left(\mathrm{x}_{\mathrm{b}}\right)$ from the ideal Bayes boundary $(\tilde{x})$, assuming $b$ is Gaussian with mean $\beta$ and variance $\sigma_{b}$, in [14] it is shown that Added Classification Error $E_{j} \propto\left(\sigma_{b}^{2}+\beta^{2}\right)$.

Mutually exclusive areas under the probability distribution are labelled $A_{1} \ldots A_{8}$ in Fig. 2, and the corresponding number of patterns for classifiers $i, j$ is given in Table I. A further assumption is that areas $\left(A_{4}, A_{5}, A_{6}, A_{7}\right)$ contain approximately equal number of $\omega_{1}$ and $\omega_{2}$ patterns, and cancel in the following calculation. By substituting from Table I into (6)

$$
s_{i j}=A_{2}+A_{8}-A_{1}-A_{3}
$$

or we may wish to use $\approx$ in $(9)$ to reflect the above assumption. Rearranging (9)

$$
s_{i j}=2\left(A_{2}-A_{3}\right)+\left(A_{8}+A_{3}\right)-\left(A_{1}+A_{2}\right)
$$

Now $\left(A_{1}+A_{2}\right)$ and $\left(A_{8}+A_{3}\right)$ represent the patterns above the tails of the distributions and are constant. If $p_{1}$ is prior probability class $\omega_{1}$ and $\mathrm{B}$ is estimated Bayes error probability, by summing $\omega_{1}$ patterns and normalising $A_{2}+A_{1}=p_{1}-B p_{1}-B\left(1-p_{1}\right)=p_{1}-B$ where $A_{4}+A_{5}=B\left(1-p_{1}\right)$. Similarly for $\omega_{2}$ patterns $A_{8}+A_{3}=1-p_{1}-B$ so that from (10)

$$
s_{i j}=2\left(A_{2}-A_{3}\right)+1-2 p_{1}
$$

From Fig. 2, the Added Classification Error of ith and jth classifiers is shown as darkly shaded regions $\left(A_{2}, A_{3}\right)$ and the difference is given by $E_{i j}=E_{i}-E_{j}=A_{2}-A_{3}$. Now from (11)

$$
E_{i j}=E_{i}-E_{j}=0.5\left(s_{i j}-1+2 p_{1}\right)
$$

so that for equal priors $s_{i j}=2 E_{i j}$. An interpretation of (6) and (12) is that a pair of complementary classifiers will have small $E_{i j}$ and therefore small $s_{i j}$ as shown in Fig. 2. 
When classifier errors are correlated, the reduction in ensemble error depends on the correlation $\delta$ averaged over all classifier pairs [14]

$$
\frac{\bar{E}}{E}=\left(\frac{1+\delta(N-1)}{N}\right)
$$

where $\overline{\mathrm{E}}$ is the ensemble Added Error and $E$ is the average individual Added Error $\left(1 / N \sum_{i=1}^{N} E_{i}\right)$. In [13] it is shown that this model represents the well-known trade-off between accuracy and diversity [12].

\section{ENSEMBLE PRUNING}

The goal in ordered aggregation pruning is to produce an ordered sequence of base classifiers $\left\{\chi_{1}, \chi_{2} \ldots \chi_{N}\right\}$. The uth classifier $\chi_{u}$ is an index into the original classifier ordering $\{1,2 \ldots N\}$. Define $C_{u-1}$ to be the set containing first $u-1$ classifiers of the ordered sequence, so that the set representing the full ensemble is $C_{N}$. The $u t h$ classifier is chosen based on likelihood of optimal improvement of the ensemble. At the $u t h$ iteration, $C_{u}$ is created from $C_{u-1}$ by selecting the classifier $\chi_{u}$ from $C_{N} \backslash C_{u-1}$, that is from the pool of classifiers not contained in $C_{u-1}$. Methods differ in both ordering heuristic and initial ensemble selection. The ordering heuristic may use the training or validation set, but in line with the findings in [2], and to give a fair comparison, we use only the training set. Note that a more extensive study would be required to compare pruning methods using a validation set.

The ordered aggregation technique proposed in this paper is based on Walsh coefficients, (3) and (6). First order Walsh pruning (W1P) puts the classifiers in descending order according to the value of first order coefficients, using (3). Second order Walsh Pruning (W2P) utilises a threshold $N_{T}$ that specifies the number of classifiers chosen from W1P to form the initial classifier ensemble for W2P. The motivation is to begin with an accurate ensemble using W1P according to (3), and then to cluster classifiers around Bayes boundary by minimising the Added Classification Error. Fig. 2 shows that a pair of classifiers with low second order coefficients are complementary with respect to the Bayes boundary. Therefore $\chi_{u}$ is chosen according to the minimum sum as follows

$$
\chi_{u}=\underbrace{\arg \min }_{j}\left(\sum_{i=1}^{u-1} s_{i j}\right)
$$

where $j \in C_{N} \backslash C_{u-1}$. In (14) the classifier is chosen that minimises the mean second order coefficients over existing classifiers. Let $W 1 P(p)$ be $\mathrm{W} 1 \mathrm{P}$ ordering for the first $p$ classifiers, and $W 2 P(p, u)$ the $\mathrm{W} 2 \mathrm{P}$ ordering with threshold $u$ for first $p$ classifiers. The optimal threshold $N_{T}$ is chosen, using the training set, based on minimum estimated training error rate, as shown in the pseudo-code in Fig, $1 . W 2 P(p, u)$ enables a different threshold $N_{T}(p)$ to be selected, depending on $p$. Since error estimates are noisy, and non-linear versus number of classifiers, second degree polynomials are used as a fitting function for error rates, although polynomial degree was not found to be critical.
Input: -W1P ordering using (3)in descending order -pruning range parameters pmin, pmax, pstep

$W 2 P(p, u)$ ordering for $p$ classifiers and fixed threshold $u$ 1) cands $=W 1 P(N) \quad \%$ candidate classifiers in W1P order 2) $W 2 P(1: u-1, u)=W 1 P(1: u-1) \quad \%$ initial ensemble $u-1$ classifiers 3) for $k=u: p$

$$
\begin{aligned}
& \text { for } j j=k: p \\
& j=\text { cands }(j j) \\
& \text { for } i i=1: k-1 \\
& \quad i=\text { cands }(i i) \\
& \quad S 2(i i)=s_{i j} \\
& \quad \text { end } \\
& \quad S 2 m(j j)=\text { mean }(S 2) \\
& \text { end }
\end{aligned}
$$

\section{Optimal threshold $N_{T}$ and error rates}

Using training set

for $p=$ pmin;pstep:pmax

1) for $u$ = pmin: $p$ compute error $W 1 P(p), W 2 P(p, u)$, end

2) fit $2^{\text {nd }}$ degree polynomials to errors $W 1 P^{*}$ and $W 2 P^{*}$

3) if $\min \left(W 2 P^{*}\right)<\min \left(W 1 P^{*}\right) \quad N_{T}(p)=\operatorname{index}\left(\min \left(W 2 P^{*}\right)\right)$

end else $N_{T}(p)=p \max \quad$ end

Using test set

for $p=$ pmin:pstep:pmax compute error $W 2 P\left(p, N_{T}(p)\right), W 1 P(p)$ end

Fig. 1: Algorithm for threshold $\mathrm{N}_{\mathrm{T}}$ and test error W1P, W2P

The computation of the Walsh coefficients in (3) and (6). has time complexity $O\left(N^{2} \cdot \mu\right)$ and may be pre-computed and stored as a symmetric $N x N$ matrix, with the first order on the diagonal and second-order off-diagonal. The time complexity of the re-ordering process defined by W2P for fixed $u$ in Fig. 1 is $O\left(N^{2}\right)$, the same as the other ordered aggregation pruning methods, as given in [2].

MDP (MDSQ in [2]) is based on the idea of a signature vector, the $m t h$ component $c_{m}^{i}$ defined to be 1 if the $m t h$ pattern is correctly classified by the $i t h$ classifier, else -1

$$
c_{m}^{i}=2 I\left(X_{m}=\Omega_{m}\right)-1
$$

where $I(\cdot)=1$ if target label and classifier agree, otherwise 0 . From (15), the $m t h(m=1 \ldots \mu)$ component of the average ensemble signature vector $c$ is defined as $\frac{1}{N} \sum_{i=1}^{N} c_{m}^{i}$, which is the margin of the mth pattern. A reference vector $\vec{O}$ is chosen with small positive margins, indicating correct classification of training patterns. The ordering is based on minimising the euclidean distance $d$ between $\vec{O}$ and $c$. Specifically, the classifier selected in the uth iteration $\chi_{u}$ is chosen from remaining classifiers by

$$
\chi_{u}=\underbrace{\arg \min }_{j} d\left(\vec{o}, 1 / N\left(c^{j}+\sum_{i=1}^{u-1} c^{i}\right)\right)
$$

where $j \in C_{N} \backslash C_{u-1}$ and both $\vec{o}$ and $c$ are $\mu-$ dimensional. As in [2], $\vec{O}$ is initially chosen with equal components of value 0.075 and varied according to $\sqrt{u}$. 
Reduced Error Pruning (REP) [17] initially selects the single classifier that gives lowest classification error. The ordering is then based on selecting the classifier $\chi_{u}$ for which the ensemble $C_{u}$ has lowest ensemble error, using the training set. Originally [17] REP used a backward selection step, but as in [2], it is not used in this study.

In pruning using Boosting-based ordered bagging (OBP) [18] [8], base classifiers are ordered according to their performance in boosting. At each iteration, the classifier with the lowest weighted training error is selected from the pool of classifiers. If all the classifiers have training error more than 50 percent, weights are reset. OBP was later combined with Instance-based Pruning [8] [9] but the results showed improvement over OBP in speed rather than accuracy.

\section{EXPERIMENTAL EVIDENCE}

Two-class benchmark problems are selected from [19], as shown in Table II. Note that dermo2, ecoli2, iris2, vehicl2 are multiclass and the class with most patterns is re-labelled $\omega_{1}=-1$ with remaining patterns $\omega_{2}=1$. As shown in Table II, all datasets have $p_{1}<0.5$ so that the constant in (12) has the same sign. Random perturbation of MLP base classifiers is caused by bootstrapping (sample patterns with replacement) [21] as well as different starting weights. The experiments are performed with two hundred single hiddenlayer MLP base classifiers using the Levenberg-Marquardt training algorithm. The number of training epochs is set to two, and the number of hidden nodes varied $\{2,3,4\}$. The ensemble uses majority vote and experiments are repeated fifty times and averaged. For these experiments $N=200$, pmin $=60$, pmax $=150$, pstep $=10$, defined in Fig. 1. Neither pmin nor pmax is critical, and chosen here to show where pruning gives improvement in error rate. Where significance is stated, it is based on the McNemar test at ninety-five percent confidence [20].

Bias/Variance will refer to $0 / 1$ loss function using Breiman's decomposition [21], for which Bias plus Variance plus Bayes equals the base classifier error rate. Bias is intended to capture the systematic difference with Bayes, and requires Bayes probability. Patterns are divided into two sets, the Bias set containing patterns for which the Bayes classification disagrees with the ensemble classifier and the Unbias set containing the remainder. Bias is computed using the Bias Set and Variance is computed using the Unbias Set, but both Bias and Variance are defined as the difference between the probabilities that the Bayes and base classifier predict the correct class label. The Bayes estimation is performed for 90/10 split using original features, and a Support Vector Classifier (SVC) with polynomial kernel run 100 times. The polynomial degree and regularisation constant are varied, and lowest test error is given in Table II.

Fig. 3 (a) shows mean W2P test error rate over all datasets for random 50/50 train/test split, with Bayes error subtracted, since improvements can then be compared with the error that is reducible. For 80 classifiers the error rate is within 1.3-1.7 percent of Bayes rate. Fig. 3 (b)-(f) show the mean results relative to $\mathrm{W} 2 \mathrm{P}$ for other pruning methods defined in Section III, and clearly indicate the overall trend. MDP outperforms other pruning methods except W2P. At 2 nodes and 80 classifiers, W2P error rate is 0.3 percent lower than MDP, but on individual datasets the difference is not statistically significant, except for ion. Fig. 3 (f) shows that un-pruned (UNP), that is random order, error rate is 5 percent higher than $\mathrm{W} 2 \mathrm{P}$ at 2 nodes and 80 classifiers. From Fig. 3 (c), as classifier becomes more powerful, the improvement of $\mathrm{W} 2 \mathrm{P}$ over MDP decreases.

To understand the results W2P, MDP and UNP are compared for the ion dataset in Fig. 4. Fig. 4 (a) shows the test errors and Fig. 4 (b) shows mean linear correlation coefficient over all pairs of classifiers, which may be used as a measure of diversity [12]. The Bias and Variance are shown in Figure 4 (c) (d), and it may be seen that Bias is minimised at 80 classifiers for $\mathrm{W} 2 \mathrm{P}$, while variance is increasing. Figure 4 demonstrates the accuracy/diversity trade-off as in (13), which appears to be optimal for 80 classifiers. It is evident that the base classifiers chosen by $\mathrm{W} 2 \mathrm{P}$ have decreasing correlation and therefore increasing diversity, particularly around 80 classifiers. From Fig. 4 (b) (d) classifiers chosen by W2P are more diverse than those chosen by MDP.

To demonstrate performance as number of training patterns is decreased, Fig. 5 (a) shows W2P with respect to Bayes rate for 20/80, 30/70 and 40/60 train/test splits at 2 nodes, 2 epochs. Fig. 5 (b) shows the corresponding Area under ROC for W2P-MDP. All other pruning methods had similar curve for AUC, which shows a very small and insignificant difference with W2P. For drawbacks of using AUC see [22].

Table II shows the best test error rates obtained for individual datasets using W2P, along with mean values of threshold $\mathrm{N}_{\mathrm{T}}$ for 2 nodes and 80 classifiers. To determine performance for fewer classifiers, experiments were repeated for $\mathrm{N}=100, \operatorname{pmin}=25, \operatorname{pmax}=70, p s t e p=5$, and the mean improvement of W2P over MDP at 2 nodes, 2 epochs was 0.25 percent at 35 classifiers.

\section{DISCUSSION}

An ensemble requires accurate yet diverse classifiers. The idea in this paper is to initially use first order coefficients to define an accurate set of base classifiers, and then minimise Added Error using an ordering based on second order coefficients, which is shown experimentally to increase diversity. A limitation of the model in Fig. 2 is the monotonicity assumption, but this is not explicitly used in our analysis, which allows any shape of Added Error not just triangular. However, if monotonicity is violated, it is likely that the assumption of equal number of patterns would be compromised and we would need to replace $=$ with $\approx$ in $(9)$ to (12). The quality of the approximation, and its effect on the proposed pruning method for specific datasets is the subject of future research. For the difficulties of extending the model to higher dimensions see the discussion in [23].

From the model in Fig 2, selecting classifiers that minimise Added Error is equivalent to finding complementary classifiers with respect to the Bayes boundary. Pruning based on second order Walsh coefficients (W2P) is an effective method, although experiments are limited in terms of parameter tuning, number and size of datasets, Further work is aimed at solving multi-class problems using the two-class decompositions implied by Error-Correcting Output Coding. Measures based on second order Walsh coefficients should also be suitable for incorporation into more sophisticated search strategies such as the optimisation framework in [4]. Furthermore, the method is suitable for any base classifier that makes a binary decision. MLP was chosen as base classifier in this paper to extend the previous work in [13]. 
Table I: Areas under Distribution defined in Fig. 2, showing number of class $\omega_{1}, \omega_{2}$ patterns defined in (5)

\begin{tabular}{l|llllllll}
\hline & $A_{1}$ & $A_{2}$ & $A_{3}$ & $A_{4}$ & $A_{5}$ & $A_{6}$ & $A_{7}$ & $A_{8}$ \\
& & & & & & & & \\
\hline$\omega_{1}=-1$ & $\mathrm{n}_{\mathrm{b}}$ & $\mathrm{n}_{\mathrm{a}}$ & & $\mathrm{n}_{\mathrm{b}}$ & $\mathrm{n}_{\mathrm{a}}$ & $\mathrm{n}_{\mathrm{a}}$ & $\mathrm{n}_{\mathrm{b}}$ & \\
& & & & & & & & \\
\hline$\omega_{2}=1$ & & & $\mathrm{n}_{\mathrm{c}}$ & $\mathrm{n}_{\mathrm{d}}$ & $\mathrm{n}_{\mathrm{c}}$ & $\mathrm{n}_{\mathrm{c}}$ & $\mathrm{n}_{\mathrm{d}}$ & $\mathrm{n}_{\mathrm{d}}$ \\
& & & & & & & & \\
\hline
\end{tabular}

Table II: Datasets showing \# patterns, prior probability $\omega_{1}$, \#features, estimated Bayes error, W2P error and threshold

\begin{tabular}{|l|l|l|l|l|l|l|}
\hline DATA & \#pat & $p_{1}$ & \#feat & $\%$ Bay & $\% W 2 P$ & $N_{T}$ \\
\hline cancer & 699 & .35 & 9 & 3.1 & 3.2 & 65 \\
\hline card & 690 & .45 & 15 & 12.8 & 13.9 & 76 \\
\hline credita & 690 & .45 & 14 & 14.1 & 14.2 & 76 \\
\hline diabetes & 768 & .35 & 8 & 22.0 & 23.2 & 68 \\
\hline heart & 920 & .45 & 35 & 16.1 & 17.6 & 72 \\
\hline ion & 351 & .36 & 34 & 6.8 & 9.3 & 61 \\
\hline sonar & 208 & .47 & 60 & 17.5 & 21.6 & 73 \\
\hline vote & 435 & .39 & 16 & 2.8 & 4.3 & 74 \\
\hline dermo2 & 366 & .31 & 33 & 0 & 0 & 60 \\
\hline ecoli2 & 336 & .43 & 7 & 2.1 & 3.7 & 69 \\
\hline iris2 & 150 & .33 & 4 & 0 & 0 & 60 \\
\hline Vehic12 & 846 & .26 & 18 & 5.0 & 6.5 & 63 \\
\hline
\end{tabular}

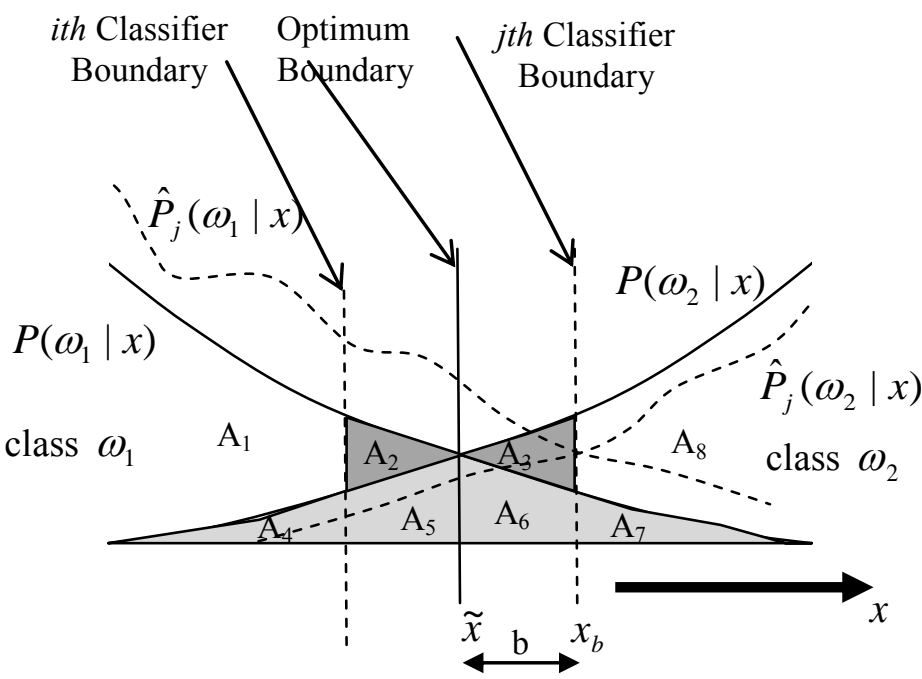

Figure 2: Model showing optimum (Bayes) boundary, ith and $j$ th classifier boundaries, estimated boundaries for jth classifier $\hat{P}_{j}$ and Added Classification Errors: darkly shaded region area $E_{i} \approx A_{2}, E_{j} \approx A_{3}$

(a) W2P-Bayes

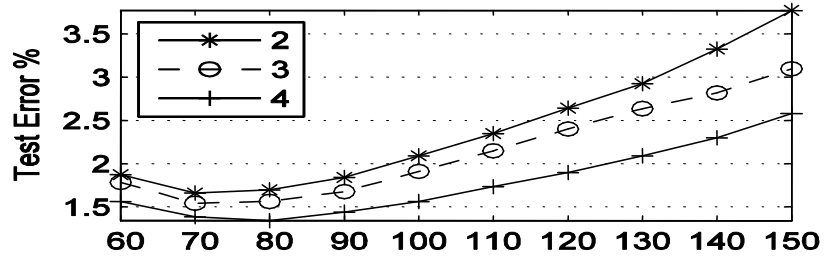

(c) MDP-W2P

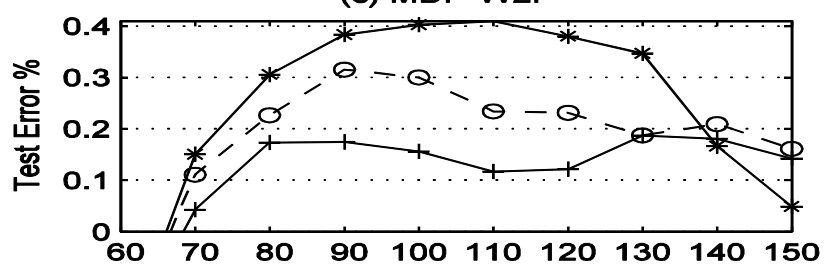

(e) REP-W2P

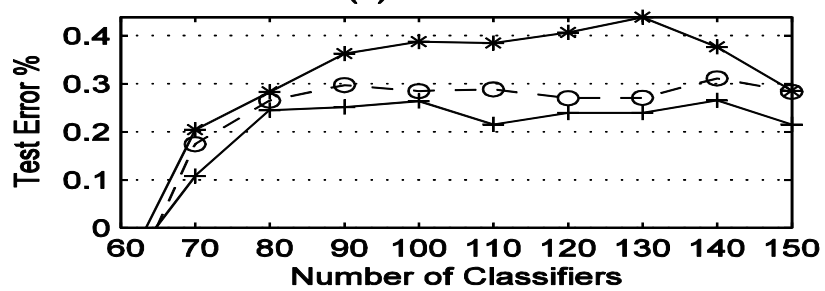

(b) OBP -W2P

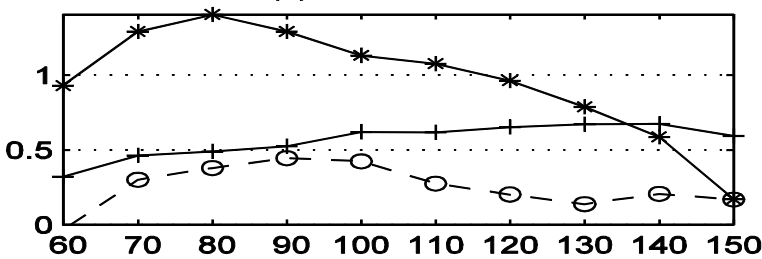

(d) W1P-W2P

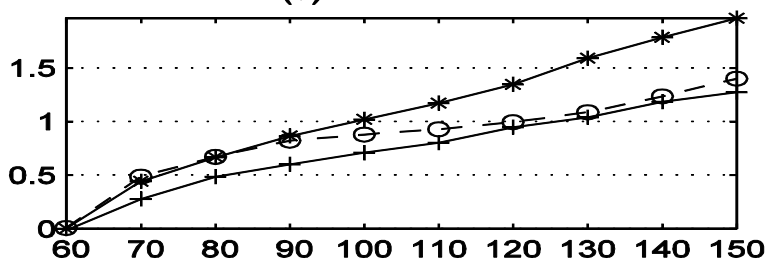

(f) UNP-W2P

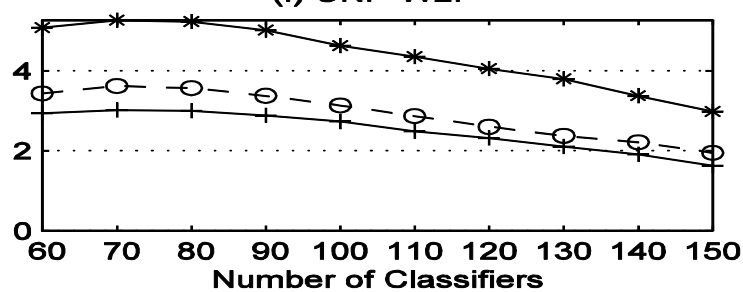

Figure 3: Mean test error rates over all datasets for 50/50 train/test split at 2 training epochs and $\{2,3,4\}$ nodes (a) W2P with Bayes estimate subtracted (b) - (f) Pruning methods minus W2P 
(a) Test Error \%

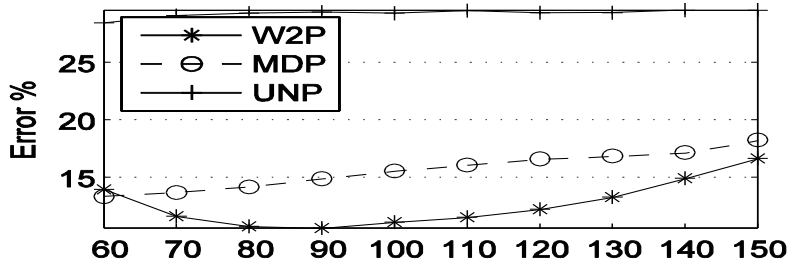

(c) Bias

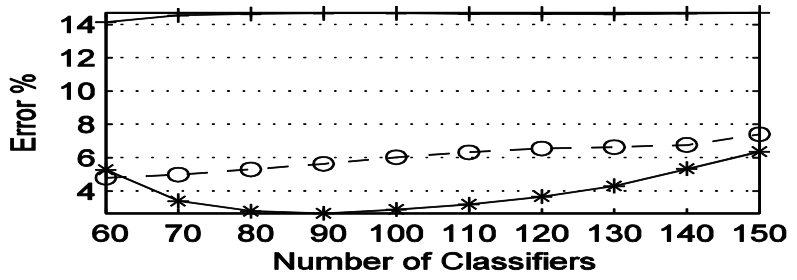

(b) Correlation

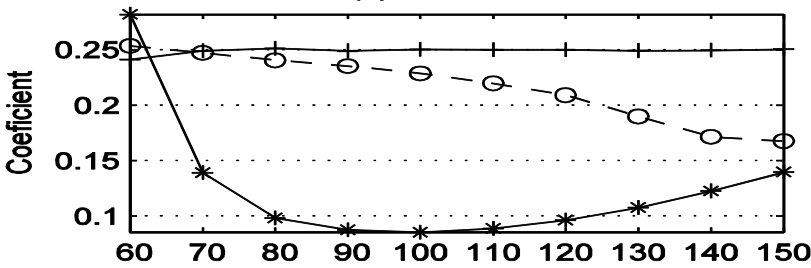

(d) Variance

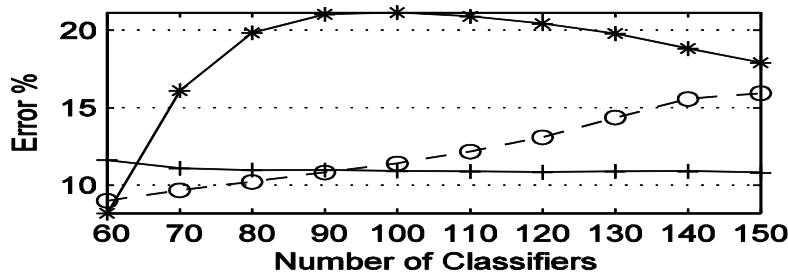

Figure 4: W2P, MDP, UNP for ion dataset at 2 epochs, 2 nodes (a) Mean test errors (b) linear correlation coefficient over all pairs classifiers (c) Bias and (d) Variance

(a) W2P-Bayes

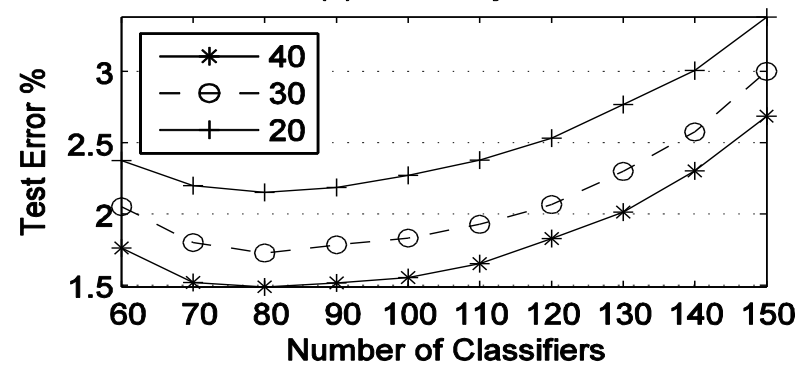

(b) W2P-MDP

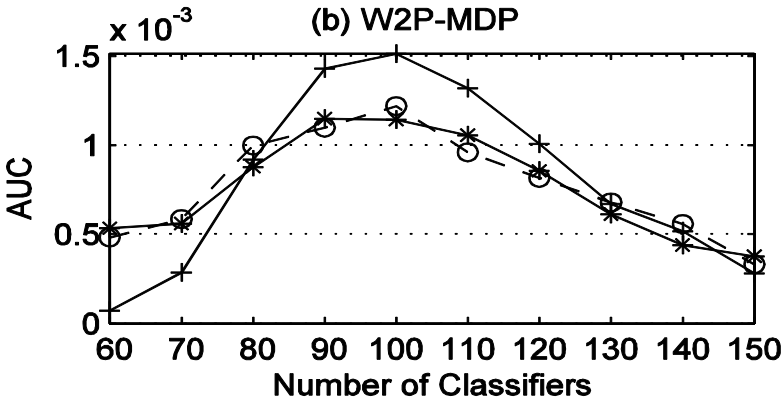

Figure 5: Mean over all datasets for [20/80,30/70,40/60] train/test split at 2 epochs, 2 nodes (a) test error rates W2P with Bayes estimate subtracted (b) Area under ROC for W2P minus MDP

\section{References}

[1] R. E. Banfield, L. O. Hall, K. W. Bowyer and W. P. Kegelmeyer, "Ensemble Diversity Measures and Their Application to Thinning", Information Fusion, vol. 6, no. 1, pp. 49-62, 2002.

[2] G. Martinez-Munoz,D. Hernandez-Lobato and A. Suarez, "An Analysis of Ensemble Pruning Techniques Based on Ordered Aggregation”, IEEE Trans. PAMI, pp. 245-259, Feb. 2009.

[3] G. Tsoumakas, I. Partalas and I. Vlahavas "An Ensemble Pruning Primer", Studies in Computational Intelligence, vol. 245, pp. 1-13, 2009.

[4] Y. Zhang, S. Burer and W.N. Street, "Ensemble Pruning via Semi-definite Programming", Journal of Machine Learning Research, vol. 7, pp. 1315-1338, 2006.

[5] H. Chan, P. Tino and X. Yao, "Predictive Ensemble Pruning by Expectation Propagation", IEEE Trans. Knowledge and Data Engineering, vol. 2, no. 6, pp. $999-1013,2009$.

[6] Z. Xie, Y. Xu, Q, Hu and P. Zhu, "Margin Distribution Based Bagging Pruning", Neurocomputing, vol. 85, pp. 11-19, 2012.

[7] M. Islam, A. Sattar, F. Amin, X. Yao and K. Murase, "A New Adaptive Merging and Growing Algorithm for Designing Artificial Neural Networks", IEEE Trans Systems, Man and Cyb., PartB: Cybernetics, vol. 39, no. 3, pp. 705-722, 2009.

[8] D. Hernandez-Lobato, G. Martinez-Munoz and A. Suarez, "Statistical Instance-based Pruning in Ensembles of Independ. Classifiers", IEEE Trans PAMI, pp. 364-369, Feb. 2009.

[9] V. Soto, D. Hernandez-Lobato, G. Martinez-Munoz and A Suarez, "A Double Pruning Algorithm for Classification Ensembles", Proc. of 9th Workshop on Multiple Classifier Systems, Cairo, Egypt, Apr. 2010, pp. 104-113.

[10] L. Hurst, D. M. Miller and J. Muzio, Spectral Techniques in Digital Logic, Academic Press, 1985.

[11] T. Windeatt, "Vote Counting Measures for Ensemble Classifiers", Pattern Recognition, vol. 36, no. 12, pp. 2743-2756, 2003.
[12] T. Windeatt, "Accuracy/Diversity and Ensemble Classifier Design”, IEEE Trans Neural Networks, vol. 17 no.5, pp.11941211, 2006.

[13] T. Windeatt and C. Zor, "Minimising Added Classification Error using Walsh Coefficients", IEEE Trans Neural Networks, vol. 22 no. 8, pp. 1334- 1339, 2011.

[14] K. Tumer and J. Ghosh, "Error Correlation and Error Reduction in Ensemble Classifiers", Connection Science, vol. 8 no. 3, pp. 385-404, 1996.

[15] B. J. Falkowski and M.A.Perkowski, "Effective Computer Methods for the Calculation of Rademacher-Walsh Spectrum for Completely and Incompletely Specified Boolean Functions," IEEE Trans. on Computer-Aided Design, vol. 11, pp.1207-1226, Oct. 1992.

[16 ] K. Tumer and J. Ghosh, "Analysis of Linear Decision Boundaries in Linearly Combined Neural Classifiers", Pattern Recognition, vol. 29, no. 2, pp. 341-348, 1996.

[17] D.D. Margineantu and T. G. Dietterich, "Pruning Adaptive Boosting", Proc. of $14^{\text {th }}$ International Conference on Machine Learning, Nashville, USA, July 1997, pp. 211-218.

[18] G. Martinez-Munoz and A. Suarez, "Using Boosting to Prune Bagging Ensembles", Pattern Recognition Letters, vol. 28, pp. 156-165, 2007.

[19] C. J. Merz and P. M. Murphy, UCI repository of ML database [Online], http://www.ics.uci.edu/ mlearn/MLRepository.html

[20] T. G. Dietterich, "Approx. statistical tests for comparing supervised classification learning algorithms", Neural Computation, vol. 10 no. 7, pp. 1895-1923, 1998.

[21] L. Breiman, "Arcing Classifiers", The Annals of Statistics, vol. 26, no. 3, pp. 801-849, 1998.

[22 ] D. J. Hand, "Measuring classifier performance: a coherent alternative to the Area under the ROC curve", Machine Learning, vol. 77, no. 1, pp. 103-123, 2009.

[23 ] L. I. Kuncheva, Combining Pattern Classifiers, Wiley, 2004. 